\title{
A conjecture concerning the exponential diophantine equation $a^{x}+b^{y}=c^{z}$
}

\author{
by \\ MaOHua Le (Zhanjiang and Shanghai)
}

1. Introduction. Let $\mathbb{Z}, \mathbb{N}, \mathbb{Q}$ be the sets of all integers, positive integers and rational numbers respectively. Let $a, b, c$ be fixed coprime positive integers with $\min (a, b, c)>1$. In 1933, Mahler [10] used his $p$-adic analogue of the method of Thue-Siegel to prove that the equation

$$
a^{x}+b^{y}=c^{z}, \quad x, y, z \in \mathbb{Z},
$$

has only finitely many solutions $(x, y, z)$. His method is ineffective. An effective result for solutions of (1) was given by Gel'fond [3]. In 1994, Terai [12] conjectured that if $(1)$ has a solution $(x, y, z)=(p, q, r)$ with $\min (p, q, r)>1$, then (1) has only one solution. In 1999, Cao [1] showed that Terai's conjecture is clearly false. He suggested that the condition $\max (a, b, c)>7$ should be added to the hypotheses of the conjecture. He used the term "Terai-Jeśmanowicz conjecture" for the resulting statement. However, the Terai-Jeśmanowicz conjecture is also false. For example, if $a=2, b=$ $2^{n}-1, c=2^{n}+1$, where $n$ is a positive integer with $n>2$, then $a, b, c$ satisfy $\max (a, b, c)>7$ and $a^{n+2}+b^{2}=c^{2}$, but (1) has two solutions $(x, y, z)=(1,1,1)$ and $(n+2,2,2)$. This implies that there exist infinitely many counterexamples to the Terai-Jeśmanowicz conjecture. On the other hand, heuristics indicate that the following statements are true.

Conjecture. The equation (1) has at most one solution $(x, y, z)$ with $\min (x, y, z)>1$.

The above mentioned conjecture was first proposed by the author [6] for primes $a, b$ and $c$. It was proved for some special cases. But, in general, the problem has not been solved yet.

2000 Mathematics Subject Classification: 11D61, 11J86.

Supported by the National Natural Science Foundation of China (No. 10271104), the Guangdong Provincial Natural Science Foundation (No. 011781) and the Natural Science Foundation of the Education Department of Guangdong Province (No. 0161). 
Most of the results concerning the above conjecture deal with the case that (1) has the solution $(x, y, z)=(2,2, r)$, where $r$ is an odd integer with $r>1$ (see [1], [7]-[9], [12]-[16]). Then from $(1)$ we get $2 \nmid c$ and $a \not \equiv b(\bmod 2)$. We may assume that $2 \nmid a$ and $2 \mid b$. Thus, $a, b$ and $c$ satisfy

$$
a^{2}+b^{2}=c^{r}, \quad \operatorname{gcd}(a, b)=1,2 \nmid a, 2 \mid b, r>1,2 \nmid r .
$$

In this connection, Terai [15] proved that if $a \geq 41 b, a \equiv 3(\bmod 8), b \equiv 2$ $(\bmod 4)$ and $(b / a)=-1$, where $(* / *)$ denotes the Jacobi symbol, then (1) has only the solution $(x, y, z)=(2,2, r)$. In this paper we prove the following general result:

TheOREM. Let $a, b, c$ be positive integers satisfying (2). If $a>b, a \equiv 3$ $(\bmod 4), b \equiv 2(\bmod 4)$ and

$$
a / b>\left(e^{r / 1856}-1\right)^{-1 / 2},
$$

then (1) has only the solution $(x, y, z)=(2,2, r)$.

Since $r \geq 3$, our theorem has the following immediate corollary.

Corollary. Let $a, b, c$ be positive integers satisfying (2). If $a>b$, $a \equiv 3(\bmod 4), b \equiv 2(\bmod 4)$ and either $a \geq 25 b$ or $r \geq 1287$, then $(1)$ has only the solution $(x, y, z)=(2,2, r)$.

\section{Preliminaries}

LEMMA 1 ([11, pp. 12-13]). Every solution $(X, Y, Z)$ of the equation

$$
X^{2}+Y^{2}=Z^{2}, \quad X, Y, Z \in \mathbb{N}, \operatorname{gcd}(X, Y)=1,2 \mid Y,
$$

can be expressed as

$$
X=A^{2}-B^{2}, \quad Y=2 A B, \quad Z=A^{2}+B^{2},
$$

where $A, B$ are positive integers satisfying

$$
A>B, \quad \operatorname{gcd}(A, B)=1,2 \mid A B .
$$

Lemma 2 ([11, pp. 122-123]). Let $n$ be a positive integer with $n>1$. Then every solution $(X, Y, Z)$ of the equation

$$
X^{2}+Y^{2}=Z^{n}, \quad X, Y, Z \in \mathbb{N}, \operatorname{gcd}(X, Y)=1,2 \mid Y,
$$

can be expressed as

$$
\begin{aligned}
& X=\left|\sum_{i=0}^{[n / 2]}\left(\begin{array}{c}
n \\
2 i
\end{array}\right) A^{n-2 i}\left(-B^{2}\right)^{i}\right|, \\
& Y=B\left|\sum_{i=0}^{[(n-1) / 2]}\left(\begin{array}{c}
n \\
2 i+1
\end{array}\right) A^{n-2 i-1}\left(-B^{2}\right)^{i}\right|, \\
& Z=A^{2}+B^{2},
\end{aligned}
$$

where $A, B$ are positive integers satisfying $\operatorname{gcd}(A, B)=1$ and $2 \mid A B$. 
Lemma 3 ([11, Theorem 4.1]). The equation

$$
X^{4}+Y^{4}=Z^{2}, \quad X, Y, Z \in \mathbb{N}, \operatorname{gcd}(X, Y)=1,
$$

has no solutions $(X, Y, Z)$.

Lemma 4 ([11, Theorem 4.2]). The equation

$$
X^{4}-Y^{4}=Z^{2}, \quad X, Y, Z \in \mathbb{N}, \operatorname{gcd}(X, Y)=1,
$$

has no solutions $(X, Y, Z)$.

LeMma 5 ([2]). Let $n$ be a positive integer with $n \geq 3$. The equation (11) $X^{n}+Y^{n}=2 Z^{n}, \quad X, Y, Z \in \mathbb{N}, \operatorname{gcd}(X, Y, Z)=1, X Y Z \neq 0$ or \pm 1 , has no solutions $(X, Y, Z)$.

Lemma 6 ([4, Theorems 6.7.1 and 6.7.4]). For any positive integer $k$ such that $k>1$ and $4 \nmid k$, let

$$
V(k)=\prod_{p \mid k}(1+\psi(p)),
$$

where $p$ runs over distinct prime divisors of $k$, and

$$
\psi(p)= \begin{cases}0 & \text { if } p=2, \\ (-1)^{(p-1) / 2} & \text { if } p \neq 2 .\end{cases}
$$

Then the equation

$$
X^{2}+Y^{2}=k, \quad X, Y \in \mathbb{N}, \operatorname{gcd}(X, Y)=1,
$$

has exactly $4 V(k)$ solutions $(X, Y)$.

Lemma 7. If $a<31 b, c \equiv 5(\bmod 8)$ and $c$ is not a prime power, then $b>58$.

Proof. Since $a<31 b$, we deduce from (2) that $926 b^{2}>c^{r}$. Hence, if $c^{r}>926.58^{2}=3236168$, then $b>58$. On the other hand, we see from (2) that every prime divisor $p$ of $c$ satisfies $p \equiv 1(\bmod 4)$. Therefore, $c^{r}=85^{3}$ is the unique integer such that $2 \nmid r, r \geq 3, c \equiv 5(\bmod 8), c^{r}<3236168$ and $c$ is not a prime power. By Lemma 6 , the equation

$$
A^{2}+B^{2}=85, \quad A, B \in \mathbb{N}, \operatorname{gcd}(A, B)=1,2 \mid B,
$$

has exactly two solutions $(A, B)=(7,6)$ and $(9,2)$. Therefore, by Lemma 2 , $(a, b)=(413,666)$ and $(621,478)$ are the only positive integers satisfying (2). Thus, the lemma is proved.

Lemma $8([1$, Theorem $])$. If $a \equiv 3(\bmod 4), c \equiv 5(\bmod 5)$ and $c$ is $a$ prime power, then (1) has only the solution $(x, y, z)=(2,2, r)$.

Lemma 9. If $a>b, a \equiv 3(\bmod 4), b \equiv 2(\bmod 4)$ and $(x, y, z)$ is $a$ solution of (1) with $(x, y, z) \neq(2,2, r)$, then $2 \mid x, x \geq 6, y=2$ and $2 \nmid z$. 
Proof. Let $(x, y, z)$ be a solution of (1) with $(x, y, z) \neq(2,2, r)$. By Lemma 2, we see from (2) that

$$
\begin{aligned}
& a=u\left|\sum_{i=0}^{(r-1) / 2}\left(\begin{array}{c}
r \\
2 i
\end{array}\right) u^{r-2 i-1}\left(-v^{2}\right)^{i}\right|, \\
& b=v\left|\sum_{i=0}^{(r-1) / 2}\left(\begin{array}{c}
r \\
2 i+1
\end{array}\right) u^{r-2 i-1}\left(-v^{2}\right)^{i}\right|, \\
& c=u^{2}+v^{2}, \quad u, v \in \mathbb{N}, \operatorname{gcd}(u, v)=1,2 \mid v .
\end{aligned}
$$

Since $b \equiv 2(\bmod 4)$, we see from $(13)$ that $v \equiv 2(\bmod 4)$ and $c \equiv 5(\bmod 8)$. Therefore, by [1, Lemma 3], we get $2 \mid y$. On the other hand, since $a \equiv 3$ $(\bmod 4)$, we deduce from $(1)$ that $q^{x} \equiv 3^{x} \equiv c^{z}-b^{y} \equiv 1(\bmod 4)$. This implies that $2 \mid x$.

Since $(x, y, z) \neq(2,2, r)$, if $y=2$, then $x \geq 4$ and $z>r$. When $x=4$, we find from (1) and (2) that

$$
a^{2}\left(a^{2}-1\right)=c^{r}\left(c^{z-r}-1\right) .
$$

Since $\operatorname{gcd}(a, c)=1$, by $(14)$, we get $a^{2}-1 \equiv 0\left(\bmod c^{r}\right)$. Hence we obtain $c^{r}=a^{2}+b^{2}>a^{2}-1 \geq c^{r}$, which is a contradiction. So we have $x \geq 6$. Further, since $c^{z} \equiv a^{x}+b^{2} \equiv 5(\bmod 8)$, we get $2 \nmid z$. Thus, if $y=2$, then $2 \mid x, x \geq 6$ and $2 \nmid z$.

If $y=4$, then $c^{z} \equiv a^{x}+b^{4} \equiv 1(\bmod 8)$, whence we get $2 \mid z$. Hence, by Lemma 3, we see from (1) that $4 \nmid x$. When $x=2$ or 6 , we infer from (1) and (2) that either $b^{2}-1 \equiv 0\left(\bmod c^{r}\right)$ or $a^{2}+1 \equiv 0\left(\bmod c^{r}\right)$. However, since $c^{r}=a^{2}+b^{2}>\max \left(b^{2}-1, a^{2}+1\right)$ that is impossible. So we have $x \geq 10$. On the other hand, by Lemma 1, we find from (1) that

$$
a^{x / 2}=A^{2}-B^{2}, \quad b^{2}=2 A B, \quad c^{z / 2}=A^{2}+B^{2},
$$

where $A, B$ are positive integers satisfying (6). Since $a>b$, we deduce from (15) that $A^{2}>a^{x / 2} \geq a^{5}>b^{5}=(2 A B)^{5 / 2}>A^{2}$, a contradiction. So we have $y \neq 4$.

Similarly, if $y>4$, then we have $2 \mid z$ and

$$
a^{x / 2}=A^{2}-B^{2}, \quad b^{y / 2}=2 A B, \quad c^{z / 2}=A^{2}+B^{2} .
$$

Since $y / 2 \geq 3$, we see from $(16)$ that $4 \mid A B$ and $c^{z / 2} \equiv 1(\bmod 8)$. This implies that $4 \mid z$. Hence, by Lemma 4 , we get $4 \nmid y$. Since $(X, Y, Z)=$ $\left(a^{x / 2}, b^{y / 2}, c^{z / 4}\right)$ is a solution of (7) for $n=4$, Lemma 2 yields

$$
\begin{gathered}
a^{x / 2}=\left|X_{1}^{4}-6 X_{1}^{2} Y_{1}^{2}+Y_{1}^{4}\right|, \quad b^{y / 2}=4 X_{1} Y_{1}\left(X_{1}^{2}-Y_{1}^{2}\right), \\
c^{z / 4}=X_{1}^{2}+Y_{1}^{2},
\end{gathered}
$$

where $X_{1}, Y_{1}$ are positive integers satisfying $X_{1}>Y_{1}, \operatorname{gcd}\left(X_{1}, Y_{1}\right)=1$ and 
$2 \mid X_{1} Y_{1}$. From (17), we obtain

$$
\begin{gathered}
X_{1}+Y_{1}=b_{1}^{y / 2}, \quad X_{1}-Y_{1}=b_{2}^{y / 2}, \\
X_{1}=\left\{\begin{array}{ll}
b_{3}^{y / 2}, \\
b_{4}^{y / 2} / 4,
\end{array} Y_{1}= \begin{cases}b_{4}^{y / 2} / 4 & \text { if } 2 \nmid X_{1}, \\
b_{3}^{y / 2} & \text { if } 2 \mid X_{1},\end{cases} \right.
\end{gathered}
$$

where $b_{1}, b_{2}, b_{3}, b_{4}$ are positive integers satisfying $b_{1} b_{2} b_{3} b_{4}=b, 2 \nmid b_{1} b_{2} b_{3}$ and $2 \mid b_{4}$. By (18), we get

$$
2 b_{3}^{y / 2}= \begin{cases}b_{1}^{y / 2}+b_{2}^{y / 2} & \text { if } 2 \nmid X_{1}, \\ b_{1}^{y / 2}-b_{2}^{y / 2} & \text { if } 2 \mid X_{1} .\end{cases}
$$

Since $y / 2$ is an odd integer with $y / 2 \geq 3$, Lemma 5 shows that (19) is impossible. To sum up, the lemma is proved.

LEMMA 10. Let $a_{1}, a_{2}, b_{1}, b_{2}$ be positive integers, and let $\Lambda=b_{1} \log a_{1}-$ $b_{2} \log a_{2}$. If $a_{1} \geq 85, a_{2} \geq 553$ and $\Lambda \neq 0$, then

$$
\log |\Lambda|>-17.17\left(\log a_{1}\right)\left(\log a_{2}\right)(1.7735+B)^{2},
$$

where

$$
B=\max \left(8.445,0.2257+\log \left(\frac{b_{1}}{\log a_{2}}+\frac{b_{2}}{\log a_{1}}\right)\right) .
$$

Proof. For any real number $\varrho$ with $\varrho>1$, by [5, Théorème 2$]$, we have (22) $\log |\Lambda|$

$$
\begin{aligned}
\geq & -\frac{16 A_{1} A_{2}}{9 \lambda^{3}}\left(B+\lambda+\frac{\lambda^{2}}{4 B}\right)^{2}\left(1+\frac{3}{2} \lambda^{3}\left(A_{1}^{-1}+A_{2}^{-1}\right)\left(B+\lambda+\frac{\lambda^{2}}{4 B}\right)^{-1}\right. \\
& +\sqrt[3]{2} \lambda^{3 / 2}\left(A_{1} A_{2}\left(B+\lambda+\frac{\lambda^{2}}{4 B}\right)\right)^{-1 / 2}+\frac{9 \lambda^{3}}{8 A_{1} A_{2}}\left(B+\lambda+\frac{\lambda^{2}}{4 B}\right)^{-1} \\
& \left.+\frac{9 \lambda^{3}}{16 A_{1} A_{2}}\left(B+\lambda+\frac{\lambda^{2}}{4 B}\right)^{-2} \log \frac{A_{1} A_{2}(B+\lambda)^{2}}{\lambda^{2}}\right)+\frac{\lambda}{2}+\log \lambda-0.15,
\end{aligned}
$$

where $\lambda=\log \varrho$ and $A_{1}, A_{2}, B$ satisfy $A_{j} \geq \max \left(2,2 \lambda,(\varrho+1) \log a_{j}\right)(j=$ $1,2)$,

$$
B \geq \max \left(5 \lambda, 1.56+\log \lambda+\log \left(\frac{b_{1}}{A_{2}}+\frac{b_{2}}{A_{1}}\right)\right) .
$$

We now choose $\varrho=e^{1.689}$ and $B$ as in $(21)$. Then we have $\lambda=1.689, A_{j}>$ $6.414 \log a_{j}(j=1,2)$ and $B$ satisfies (23). Since $B \geq 8.445$, we get $B+\lambda+$ $\lambda^{2} /(4 B)>B+1.7735 \geq 10.2185$. Therefore, if $a_{1} \geq 85$ and $a_{2} \geq 553$, then

$$
\frac{3}{2} \lambda^{3}\left(A_{1}^{-1}+A_{2}^{-1}\right)\left(B+\lambda+\frac{\lambda^{2}}{4 B}\right)^{-1}<0.04264,
$$




$$
\begin{aligned}
& \sqrt[3]{2} \lambda^{3 / 2}\left(A_{1} A_{2}\left(B+\lambda+\frac{\lambda^{2}}{4 B}\right)\right)^{-1 / 2}<0.05745 \\
& \frac{9 \lambda^{3}}{8 A_{1} A_{2}}\left(B+\lambda+\frac{\lambda^{2}}{4 B}\right)^{-1}<0.00047 \\
& \frac{9 \lambda^{3}}{16 A_{1} A_{2}}\left(B+\lambda+\frac{\lambda^{2}}{4 B}\right)^{-2} \log \frac{A_{1} A_{2}(B+\lambda)^{2}}{\lambda^{2}}<0.00025
\end{aligned}
$$

Since $\lambda / 2+\log \lambda-0.15>0$, we find from (22) that $(20)$ holds. Thus, the lemma is proved.

3. Proof of the Theorem. By Lemma 8, if $c$ is a prime power, then the assertion of the Theorem holds. Therefore, we may assume that $c$ is not a prime power. We observe that $c \equiv 5(\bmod 8)$ if $b \equiv 2(\bmod 4)$. As before, we see from $(2)$ that every prime divisor $p$ of $c$ satisfies $p \equiv 1(\bmod 4)$. Hence, we get $c \geq 85$.

Let $(x, y, z)$ be a solution of $(1)$ with $(x, y, z) \neq(2,2, r)$. By Lemma 9 , then we have $2 \mid x, x \geq 6, y=2$ and $2 \nmid z$. Hence, (1) can be rewritten as

$$
a^{x}+b^{2}=c^{z}, \quad 2 \mid x, x \geq 6,2 \nmid z .
$$

From (24), we get

$$
z \log c=x \log a+\theta
$$

where

$$
0<\theta=\frac{2 b^{2}}{a^{x}+c^{z}} \sum_{i=0}^{\infty} \frac{1}{2 i+1}\left(\frac{b^{2}}{a^{x}+c^{z}}\right)^{2 i}<\frac{3 b^{2}}{2 a^{x}}
$$

Let $\Lambda=z \log c-x \log a$. Since $a>b$ and $c^{r} \geq 85^{3}$, we deduce from (2) that $a>\sqrt{c^{r} / 2}>553$. Therefore, by Lemma 10, we have

$$
\log |\Lambda|>-17.17(\log c)(\log a)(1.7735+B)^{2}
$$

where

$$
B=\max \left(8.445,0.2257+\log \left(\frac{z}{\log a}+\frac{x}{\log c}\right)\right) .
$$

On the other hand, by (25) and (26), we get

$$
\log \left(3 b^{2} / 2\right)-\log |\Lambda|>x \log a .
$$

The combination of (27) and (29) yields

$$
\frac{\log \left(3 b^{2} / 2\right)}{(\log a)(\log c)}+17.17(1.7735+B)^{2}>\frac{x}{\log c}
$$


If $8.445 \geq 0.2257+\log (z / \log a+x / \log c)$, then

$$
\frac{2 x}{\log c}<\frac{z}{\log a}+\frac{x}{\log c} \leq e^{8.2193}<3712,
$$

whence we obtain

$$
\frac{x}{\log c}<1856 .
$$

Since $a>b$ and $c>84$, if $8.445<0.2257+\log (z / \log a+x / \log c)$, then from (28) and (30) we get

$$
1+17.17\left(1.9992+\log \left(\frac{2 x}{\log c}+\theta\right)\right)^{2}>\frac{x}{\log c} .
$$

Since $\theta<3 b^{2} /\left(2 a^{x}\right)<3 /\left(2 a^{x-2}\right) \leq 3 /\left(2 a^{4}\right)<10^{-10}$ by $(26)$ and $a>553$, we conclude from (33) that (32) also holds.

Let $t=a / b$. We find from (2) that

$$
r \log c=2 \log a+\log \left(1+\frac{1}{t^{2}}\right) .
$$

By (25) and (34), we obtain

$$
0<(r x-2 z) \log c=x \log \left(1+\frac{1}{t^{2}}\right)-2 \theta<x \log \left(1+\frac{1}{t^{2}}\right) .
$$

Since $2 \mid x$, we have $r x-2 z \geq 2$, and by (35), we get

$$
\frac{x}{\log c}>\frac{2}{\log \left(1+t^{-2}\right)} .
$$

The combination of (32) and (36) yields

$$
928 \log \left(1+\frac{1}{t^{2}}\right)>1
$$

whence we conclude that

$$
t<31 \text {. }
$$

On the other hand, we see from (2) and (24) that

$$
\begin{aligned}
& \left(a^{2}+b^{2}\right)^{x / 2}-\left(a^{x}+b^{2}\right) \\
& \quad=b^{2}\left(\sum_{j=1}^{x / 2}\left(\begin{array}{c}
x / 2 \\
j
\end{array}\right) a^{x-2 j} b^{2(j-1)}-1\right)=c^{z}\left(c^{r x / 2-z}-1\right) .
\end{aligned}
$$

Since $\operatorname{gcd}(b, c)=1$, we deduce from (39) that

$$
\sum_{j=1}^{x / 2}\left(\begin{array}{c}
x / 2 \\
j
\end{array}\right) a^{x-2 j} b^{2(j-1)} \equiv 1\left(\bmod c^{z}\right) .
$$


This implies that

$$
\sum_{j=1}^{x / 2}\left(\begin{array}{c}
x / 2 \\
j
\end{array}\right) a^{x-2 j} b^{2(j-1)}>c^{z}>a^{x} .
$$

Since $a>b$, we find from (2) and (41) that

$$
t^{2}\left(1+\frac{1}{t^{2}}\right)^{x / 2}>t^{2}\left(\left(1+\frac{1}{t^{2}}\right)^{x / 2}-1\right)>a^{2}>\frac{c^{r}}{2},
$$

whence we obtain

$$
\frac{x}{\log c}>\left(2 r-\frac{2 \log \left(2 t^{2}\right)}{\log c}\right) / \log \left(1+\frac{1}{t^{2}}\right) .
$$

If $r \leq\left(2 \log \left(2 t^{2}\right)\right) / \log c$, then we have

$$
\frac{4 a^{4}}{b^{4}}=4 t^{4} \geq c^{r}=a^{2}+b^{2}>a^{2},
$$

whence $a^{2}>b^{4} / 4$ and $2 t>b$. Since $b \equiv 2(\bmod 4),(38)$ implies that $b \leq 58$. However, by Lemma 7 , this is impossible. So we have $r>\left(2 \log \left(2 t^{2}\right)\right) / \log c$ and

$$
\frac{x}{\log c}>\frac{r}{\log \left(1+t^{-2}\right)},
$$

by (43). The combination of (32) and (45) shows that (3) is false. Thus, the Theorem is proved.

Acknowledgements. The author would like to thank the referee for his valuable suggestions.

\section{References}

[1] Z. F. Cao, A note on the Diophantine equation $a^{x}+b^{y}=c^{z}$, Acta Arith. 91 (1999), 85-93.

[2] H. Darmon and L. Merel, Winding quotients and some variants of Fermat's Last Theorem, J. Reine Angew. Math. 490 (1997), 81-100.

[3] A. O. Gel'fond, Sur la divisibilité de la différence des puissances de deux nombres entiers par une puissance d'un idéal premier, Mat. Sb. 7 (1940), 7-25.

[4] L.-K. Hua, Introduction to Number Theory, Springer, Berlin, 1982.

[5] M. Laurent, M. Mignotte et Yu. Nesterenko, Formes linéaires en deux logarithmes et déterminants d'interpolation, J. Number Theory 55 (1995), 285-321.

[6] M. H. Le, On the Diophantine equation $a^{x}+b^{y}=c^{z}$, J. Changchun Teachers College Ser. Nat. Sci. 2 (1985), 50-62 (in Chinese).

[7] -, A note on the Diophantine equation $\left(m^{3}-3 m\right)^{x}+\left(3 m^{2}-1\right)^{y}=\left(m^{2}+1\right)^{z}$, Proc. Japan Acad. Ser. A Math. Sci. 73 (1997), 148-149.

[8] -, On Terai's conjecture concerning Pythagorean numbers, Bull. Austral. Math. Soc. 61 (2000), 329-334. 
[9] M. H. Le, On the exponential Diophantine equation $\left(m^{3}-3 m\right)^{x}+\left(3 m^{2}-1\right)^{y}=$ $\left(m^{2}+1\right)^{z}$, Publ. Math. Debrecen 58 (2001), 461-466.

[10] K. Mahler, Zur Approximation algebraischer Zahlen I: Über den grössten Primteiler binärer Formen, Math. Ann. 107 (1933), 691-730.

[11] L. J. Mordell, Diophantine Equations, Academic Press, London, 1969.

[12] N. Terai, The Diophantine equation $a^{x}+b^{y}=c^{z}$, Proc. Japan Acad. Ser. A Math. Sci. 70 (1994), 22-26.

[13] - , The Diophantine equation $a^{x}+b^{y}=c^{z}$, II, ibid. 71 (1995), 109-110.

[14] - The Diophantine equation $a^{x}+b^{y}=c^{z}$, III, ibid. 72 (1996), 20-22.

[15] - Applications of a lower bound for linear forms in two logarithms to exponential Diophantine equations, Acta Arith. 90 (1999), 17-35.

[16] N. Terai and K. Takakuwa, A note on the Diophantine equation $a^{x}+b^{y}=c^{z}$, Proc. Japan Acad. Ser. A Math. Sci. 73 (1997), 161-164.

Department of Mathematics

Zhanjiang Normal College

Zhanjiang, Guangdong

P.R. China
Department of Mathematics Shanghai Teachers University Guilin Road, Shanghai

P.R. China

Received on 17.9.2000

and in revised form on 22.5.2002 